\title{
GAS-SURFACE INTERACTION EFFECTS ON THE FLOWFIELD STRUCTURE OF HIGH SPEED MICROCHANNEL FLOW
}

\author{
Israel B. Sebastião, israel@lcp.inpe.br \\ Wilson F. N. Santos, wilson@lcp.inpe.br \\ Combustion and Propulsion Laboratory (LCP), National Institute for Space Research (INPE), Cachoeira Paulista-SP, 12630-000
}

\begin{abstract}
This work describes a computational study on supersonic flow in a microchannel composed of two parallel plates. Effects of incomplete surface accommodation on the flowfield structure have been investigated by employing the Direct Simulation Monte Carlo (DSMC) method in combination with the Cercignani-Lampis-Lord (CLL) gas-surface interaction model. The aim of this work is to investigate the disturbance developments due to differences in the accommodation coefficients on the lower and upper surfaces of the microchannel. The results presented highlight the sensitivity of the primary properties to changes on the gas-surface accommodation coefficients. It is found that accommodation coefficients have different influence on velocity, density, pressure and temperature for the conditions investigated.
\end{abstract}

Keywords: DSMC, Rarefied Flow, Microchannel, Gas-surface Interaction.

\section{INTRODUCTION}

Microelectromechanical systems (MEMS) have been considered as an emerging technology with a significant potential for future growth (Ho and Tai, 1996, 1998; O'Connor, 1992). Microactuators, microrefrigerators, microgenerators, micropumps, and micronozzles, examples of device microminiaturization, have become important because of their application to medical and engineering problems (Gad-el-Hak, 1999; Karniadakis et al., 2005; Sen et al., 1996; Titov et al., 2007). Despite of growing number of realized applications of MEMS in scientific and engineering devices, there is only a minimum level of understanding of the fluid dynamics and heat transfer processes in fuidic MEMS.

Many of these devices involve fluid flow through microchannels. In most microchannels, the mean free path $\lambda$ of the molecules is of the same order of magnitude of the height $H$ of the microchannel. In this fashion, the Knudsen number, $K n=\lambda / H$, is in the transition flow regime $(0.01<K n<10)$, i.e., between the continuum flow regime and the free molecular flow regime. Consequently, in such a circumstance, treating the microchannel flow as a continuum phenomenon may lead to inaccurate results.

According to the current literature, a great deal of studies (Alexeenko et al., 2003; Barber and Emerson, 2002; Cai and Boyd, 2007; Kaplan and Oran, 2002; Lilly et al., 2007; Liou and Fang, 2001; Oh et al., 1997; Sayegh et al., 2004) has been carried out in the last decades on microchannels. The major interest in these studies has gone into predicting the fluid behavior within microchannels. For the purpose of this introduction, it will be sufficient to describe only a few of these studies.

Oh et al. (1997) have investigated the Knudsen number effect in a high speed microchannel flow by employing the DSMC method. The Knudsen number effect was examined for three different $K n$ values, 0.07, 0.14 and 0.19, for a high-speed inflow by varying the channel height. Computational results provided contours of pressure, temperature, and Mach number. In addition, the analysis showed the complex interactions among oblique shocks and boundary layers.

The impact of the Knudsen number on the heat transfer of a supersonic flow in microchannels was examined by Liou and Fang (2001) by using the DSMC method. They found that the heat transfer to the isothermal wall increased significantly with the Knudsen number. They also found that the magnitude of the temperature jump at the wall increased with the Knudsen number rise.

Barber and Emerson (2002) have studied the influence of the Reynolds and Knudsen numbers on the hydrodynamic development length at the entrance of parallel plate microchannels. Numerical simulations were carried out over a range of Knudsen number covering from the continuum to slip flow regime, $0<K n<0.1$. The analysis showed that the entrance development region is almost $25 \%$ larger than that predicted by using continuum assumption.

Most of the available DSMC simulation results on microchannel flows are limited to velocity, pressure, and temperature distributions without reporting to the gas-surface interaction effects on the primary properties. In an effort to obtain further insight into the nature of the flowfield structure in a high speed microchannel flow under transitional flow conditions, a parametric study is performed on microchannels with a great deal of emphasis placed on the gas-surface interaction effects. In this scenario, the primary goal of the present account is to assess the sensitivity of the primary properties to variations on the surface accommodation coefficients experienced by microchannels composed by two parallel plates with different wall accommodation coefficients by employing the Cercignani-Lampis-Lord (CLL) model (Lord, 1991). The CLL model, which incorporates independent accommodation coefficients for the normal and tangential velocity components, is implemented into the DSMC code. A quantitative analysis of the flow is made for different normal 
and tangential accommodation coefficients. Particular attention will be paid to the flowfield properties, i.e., the sensitivity of the primary properties such as velocity, density, pressure and temperature, due to differences on the wall shear stresses, caused by different translational and rotational energies accommodation coefficients attributed to the surfaces.

\section{GAS-SURFACE INTERACTION MODEL}

Three models of gas-surface interactions may be employed in the DSMC method: (1) specular, (2) diffuse, and (3) some combination of these. In a specular reflection, molecules are reflected like a perfectly elastic sphere with reversal of the normal component of velocity and no changes on the parallel component of velocities and energy. In a diffuse reflection, the molecules are reflected equally in all directions usually with a complete thermal accommodation. The final velocity of the molecules is randomly assigned according to a half-range Maxwellian distribution determined by the wall temperature. The combination of diffuse reflection with specular reflection (Maxwell model) introduces a single parameter $f$ to indicate the fraction of those molecules reflected diffusely in a completely accommodated fashion according to a Maxwellian distribution corresponding to the wall temperature, and the remaining fraction (1- $f$ ), being assumed to reflect specularly.

The Maxwell model was followed by the introduction of accommodation coefficients that describe the degree of accommodation of the incident normal momentum, tangential momentum and kinetic energy to those of the surface. The traditional definition (Schaff and Chambre, 1958) for these coefficients is usually expressed as being,

$$
\alpha_{n}=\frac{e_{i}-e_{r}}{e_{i}-e_{w}} \quad \text { and } \quad \sigma_{t}=\frac{\tau_{i}-\tau_{r}}{\tau_{i}}
$$

where $e$ and $\tau$ refer to the average kinetic energy for the normal component of velocity, and momentum flux acting tangential to the surface, respectively; subscripts $i$ and $r$ stand for the incident and reflected components, and $w$ refers to the component that would be produced by a diffuse reflection at the temperature of the surface.

Data from many experiments show that molecules reflected or re-emitted from solid surfaces present lobular distributions under high vacuum conditions and are poorly represented by the Maxwell model. However, this model is widely used because it satisfies the principle of detailed balance or reciprocity. Detailed balance means that at equilibrium every molecular process and its inverse process must individually balance. A phenomenological model that satisfies detailed balance and has demonstrated improvement over the Maxwell model has been proposed by Cercignani and Lampis (1971)(CL model). This model is based on the definition of the accommodation coefficients $\alpha_{n}$ and $\alpha_{t}$ that represent the accommodation coefficients for the kinetic energy associated with the normal and tangential components of velocity. The CL model provides a continuous spectrum of behavior from specular reflection at one end to diffuse reflection with complete energy accommodation at the other, and produces physically realistic distributions of direction and energy re-emitted molecules. Lord (1991) has shown that the CL model is suited for the DSMC method, and described how to incorporate it into the DSMC method. The DSMC method with Lord's implementation is referred as the Cercignani-Lampis-Lord (CLL) method. Figure 1 displays a schematic comparison of the Maxwell reflection model and the CLL reflection model.

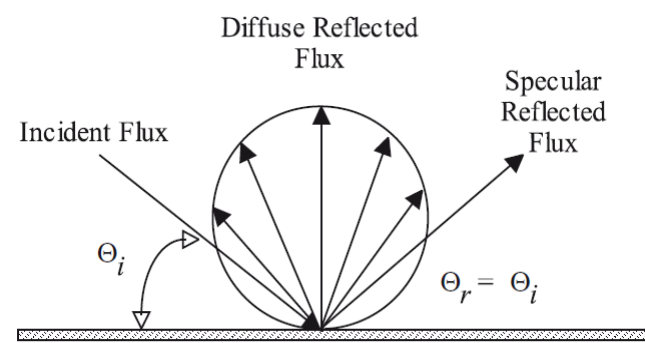

(a) Maxwell Model

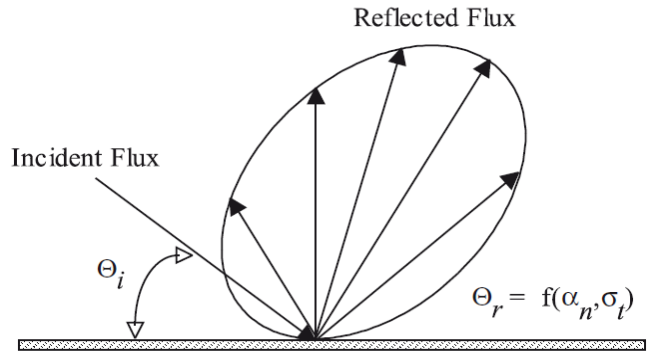

(b) CLL Model

Figure 1. Drawing illustrating (a) the Maxwell reflection model and (b) the CLL reflection model.

\section{COMPUTATIONAL METHOD}

In order to study rarefied flow with a significant degree of non-equilibrium, the Direct Simulation Monte Carlo (DSMC) method (Bird, 1994) is usually employed. The DSMC method has become the most common computational technique for modeling complex transitional flows of engineering interest. In the DSMC method, the gas flow is represented by a large number of simulated particles, where each simulated particle represents a fixed number of real gas 
particles. The simulated particles are allowed to move, to collide among themselves, and with any boundaries, while the computer stores their position coordinates, velocities and other physical properties such as internal energy. The simulation is advanced in time steps, which should be less than the mean time between collisions (Bird, 1994).

The molecular collisions are modeled by using the variable hard sphere (VHS) molecular model (Bird, 1981) and the no time counter (NTC) collision sampling technique (Bird, 1989). The energy exchange between kinetic and internal modes is controlled by the Borgnakke-Larsen statistical model (Borgnakke and Larsen, 1975). Simulations are performed using a non-reacting gas model consisting of one chemical species, $\mathrm{N}_{2}$. Energy exchanges between the translational and the internal mode of rotational is considered. Relaxation collision number of 5 was used for the calculations of rotation.

In order to simulate the partial surface accommodation, the CLL model (Lord, 1991) was implemented into this DSMC calculation. The CLL model is derived by assuming that there is no coupling between the normal and tangential momentum components. The two adjustable parameters appearing in the CLL model are the normal component of translational energy $\alpha_{n}$ and the tangential component of momentum $\sigma_{t}$. However, in the implementation of the CLL model in the DSMC method, Bird (1994) has shown that it is equivalent to specify the normal $\alpha_{n}$ and tangential $\alpha_{t}$ components of translational energy, since $\alpha_{t}=\sigma_{t}\left(2-\sigma_{t}\right)$, and thus that $\sigma_{t}<\alpha_{t}$, assuming that $\sigma_{t}$ lies between 0 and 1 . In the present simulation, $\alpha_{n}$ and $\sigma_{t}$ are used as being the two adjustable parameters. It is important to mention that in the CLL model the accommodation of internal energy $\alpha_{r}$ is allowed to be independent of the translational accommodation.

\section{COMPUTATIONAL FLOW DOMAIN AND GRID}

In order to account for particle-particle collisions, the flowfield inside the microchannels is divided into an arbitrary number of regions. The regions are further subdivided into computational cells, and the cells are subdivided into subcells. In this fashion, the cell provides a convenient reference sampling of the macroscopic gas properties, while the collision partners are selected from the same subcell for the establishment of the collision rate. A schematic view of the computational domain is depicted in Fig. 2.

According to this figure, the computational domanin was divided into five regions, from $R 1$ to $R 5$. In addition, side I is defined by the body surface. Reflection with different incomplete surface accommodation is the condition applied to upper and lower surfaces. Sides II and III are the freestream side through which simulated molecules enter and exit. Finally, vacuum condition (Bird, 1994) was defined at the downstream outflow boundary. Therefore, any simulated molecules crossing this boundary during simulation are removed, and no molecules are introduced from outside this boundary. As a result, simulated molecules can only exit.

The numerical accuracy in DSMC method depends on the cell size chosen, on the number of particles per computational cell as well as on the time step. These effects were investigated in order to determine the number of cells and the number of particles required to achieve grid independence solutions. Grid independence was tested by running the calculations with half (7,720 cells) and double (30,880 cells) the number of cells in $x$ and $y$ directions compared to a standard grid (15,440 cells) for the case base, i.e., diffuse reflection with complete accommodation, $\alpha_{n}=1$ and $\sigma_{t}=1$. In addition, each grid was made up of non-uniform cell spacing in both directions. Moreover, point clustering is used close to solid walls, and to the entrance and exit regions. The effect (not shown) of changing the cell size in both directions on the heat transfer, pressure and skin friction coefficients was rather insensitive to the range of cell spacing considered, indicating that the standard grid, with a total of 15,440 cells, for the base case, is essentially grid independent.

A similar examination was made for the number of molecules. The standard grid for the case base corresponds to, on average, a total of 323,900 molecules. Two new cases using the same grid were investigated. These two new cases correspond to 161,200 and 649,500 molecules in the entire computational domain. As the three cases presented the same results (not shown) for the heat transfer, pressure and skin friction coefficients, hence the standard grid with a total of 323,900 molecules is considered enough for the computation of the flowfield properties for the case base.

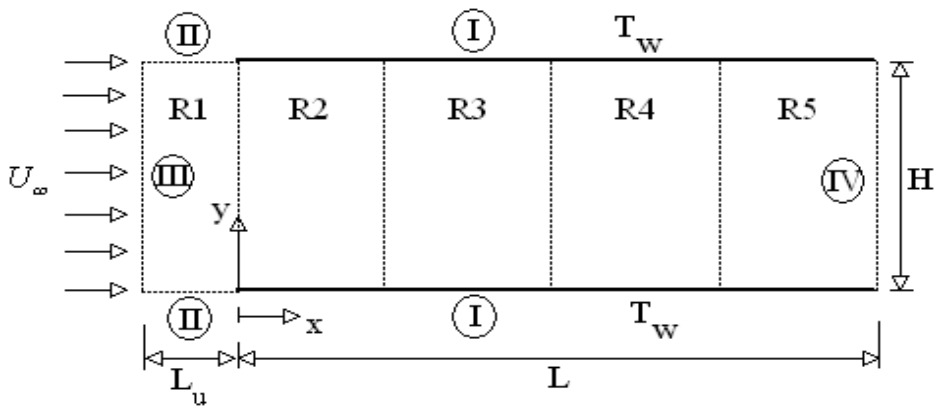

Figure 2. Schematic view of the computational domain. 


\section{FREESTREAM AND FLOW CONDITIONS}

Freestream flow conditions used for the numerical simulations are those given by Liou and Fang (2001) and Le and Hassan (2006), and summarized in Tab. 1. Based on Fig. 2, the aspect ratio of the microchannel is set at 5, which corresponds to a height $H$ of $12 \mu \mathrm{m}$ and length $L$ of $60 \mu \mathrm{m}$. Also, the freestream upstream length $L_{u}$ is $0.6 \mu \mathrm{m}$.

The freestream velocity $U_{\infty}$, assumed to be constant at $1465.5 \mathrm{~m} / \mathrm{s}$, corresponds to a freestream Mach number $M_{\infty}$ of 4.15. The translational and rotational temperatures in the freestream are in equilibrium at $300 \mathrm{~K}$. Both surfaces are maintained at a uniform temperature $T_{w}$ of $323 \mathrm{~K}$ for all cases investigated.

The overall Knudsen number $K n$ is defined as the ratio of the molecular mean free path $\lambda$ in the freestream gas to a characteristic dimension of the flowfield. In the present study, the characteristic dimension was defined as being the microchannel height $H$. Therefore, the Knudsen number $K n_{H}$ corresponds to 0.062 . Finally, the Reynolds number $R e_{H}$ is around 99.5, also based on conditions in the undisturbed stream.

In order to simulate the gas-surface interaction effects, the DSMC calculations were performed by considering the lower surface conditions defined as a diffuse reflection, $\alpha_{n}=1=\sigma_{t}$, with complete thermal accommodation, $\alpha_{r}=1$. For the upper surface, the accommodation coefficients, $\alpha_{n}$ and $\sigma_{t}$ were assumed as being $0.2,0.4,0.6,0.8$, and 1 . It should be also mentioned in this context that each value was independently set for the two coefficients.

Table 1. Freestream flow conditions

\begin{tabular}{ccccccc}
\hline$T_{\infty}(\mathrm{K})$ & $p_{\infty}\left(\mathrm{N} / \mathrm{m}^{2}\right)$ & $\rho_{\infty}\left(\mathrm{kg} / \mathrm{m}^{3}\right)$ & $\mu_{\infty}\left(\mathrm{Ns} / \mathrm{m}^{2}\right)$ & $n_{\infty}\left(\mathrm{m}^{-3}\right)$ & $\lambda_{\infty}(\mathrm{m})$ & $U_{\infty}(\mathrm{m} / \mathrm{s})$ \\
\hline 300 & $7.248 \times 10^{4}$ & 0.8138 & $1.434 \times 10^{-5}$ & $1.750 \times 10^{25}$ & $7.4 \times 10^{-8}$ & 1465.5 \\
\hline
\end{tabular}

\section{COMPUTATIONAL RESULTS AND DISCUSSION}

The purpose of this section is to discuss and to compare differences in the flowfield properties due to variations on the accommodation coefficients. Flowfield properties of particular interest in the present account are velocity, density, pressure and temperature.

\subsection{Velocity Profiles}

Streamwise velocity profiles for three cross-sections along the microchannel and their dependence on the normal and tangential accommodation coefficients are illustrated in Figs. 3 and 4, respectively. In this set of plots, velocity ratio stands for the streamwise velocity $u$ normalized by the freestream velocity $U_{\infty}, X$ is the length $x$ normalized by the microchannel length $L$, and $Y$ is the height $y$ normalized by the microchannel $H$. In addition, for comparison purpose, the velocity profiles obtained by Liou and Fang (2001), Le and Hassan (2006) and Le and Esmail (2007) are also presented.

Looking first to Fig. 3, it is seen that the boundary layer develops downstream along the microchannel; the velocity profiles are basically flat at the entrance and become parabolic for sections $X$ of 0.4 and 0.6 , indicating that the flow is fully developed. Moreover, the slip velocity at the wall is very large near the leading edges of the two plates. It is also observed that the maximum value for the velocity ratio at the centerline decreases significantly downstream along the microchannel. This is an expected behavior in the sense that a supersonic flow is decelerated in a constant-area duct as friction is taken into account. Therefore, this behavior is consistent with the Fanno solution (Shapiro, 1954).

Of particular interest is the behavior of the normal accommodation coefficient. No appreciable changes are observed in the streamwise velocity profiles due to variations on the normal accommodation coefficient $\alpha_{n}$. The reason for that is because $\alpha_{n}$ adjusts only the normal component of velocity associated with molecules reflected from the wall. Although $\alpha_{n}$ has demonstrated a weak influence on tangential velocity $u$, a strong influence on wall pressure and heat transfer distributions is expected along the microchannel surface, since both properties rely on the normal momentum exchange between wall and molecules.

Still referring to Fig. 3, it is very encouraging to observe that for the diffuse reflection case, good agreement is obtained as the velocity profiles are compared with those presented by Le and Hassan (2006) and Le and Esmail (2007). Nevertheless, at section $X=0.0$, a small difference in the velocity profiles is observed as the solution is compared with DSMC results reported by Liou and Fang (2001). It should be mentioned in this context that DSMC calculations presented by Liou and Fang (2001) correspond to a mesh with 6,000 cells, with a total of 11,854 simulated molecules. In addition, Le and Esmail (2007) used with the computational domain, also defined by 6,000 cells, 114,300 simulated molecules. In contrast, the study at hand considered a mesh with 15,440 cells, and a total of 323,900 simulated molecules. Therefore, since a large number of molecules was considered in the present account, then it is reasonable to believe that these results are more realistic that those presented by Liou and Fang (2001).

Turning next to Fig. 4, it is clearly noticed that variations on the tangential accommodation coefficient $\sigma_{t}$ affect the 

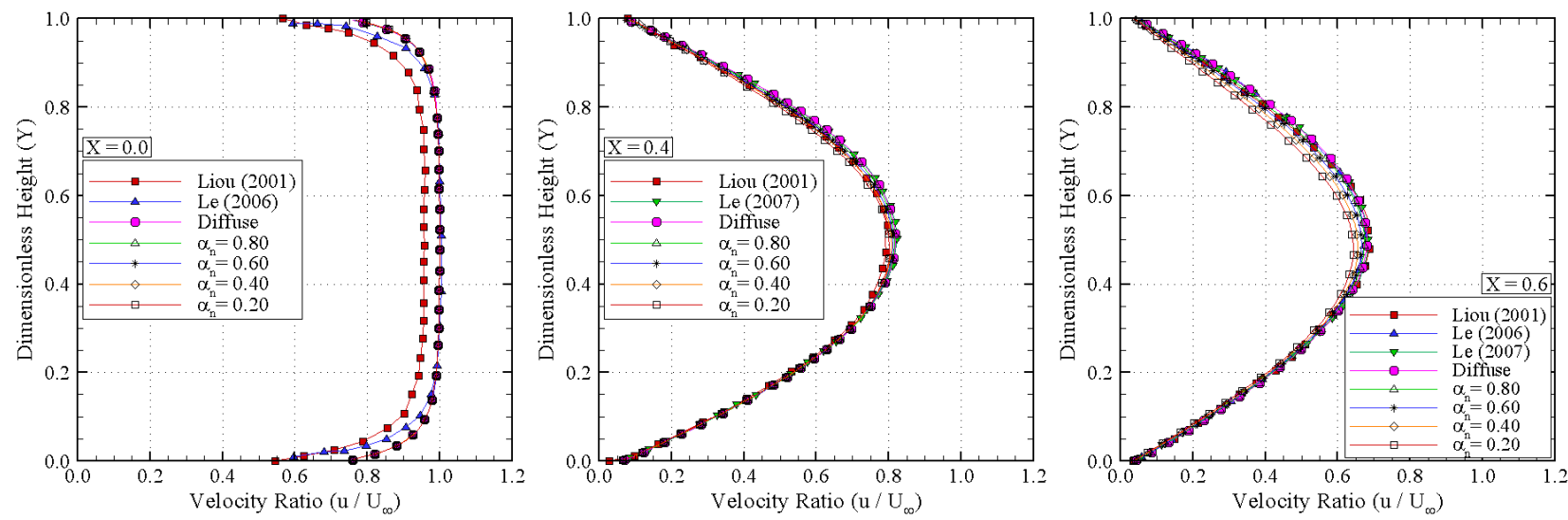

Figure 3. Velocity ratio $\left(u / U_{\infty}\right)$ profiles along the microchannel as a function of the normal accommodation coefficient $\alpha_{n}$ for section $X$ of 0.0 (left), 0.4 (middle) and 0.6 (right).
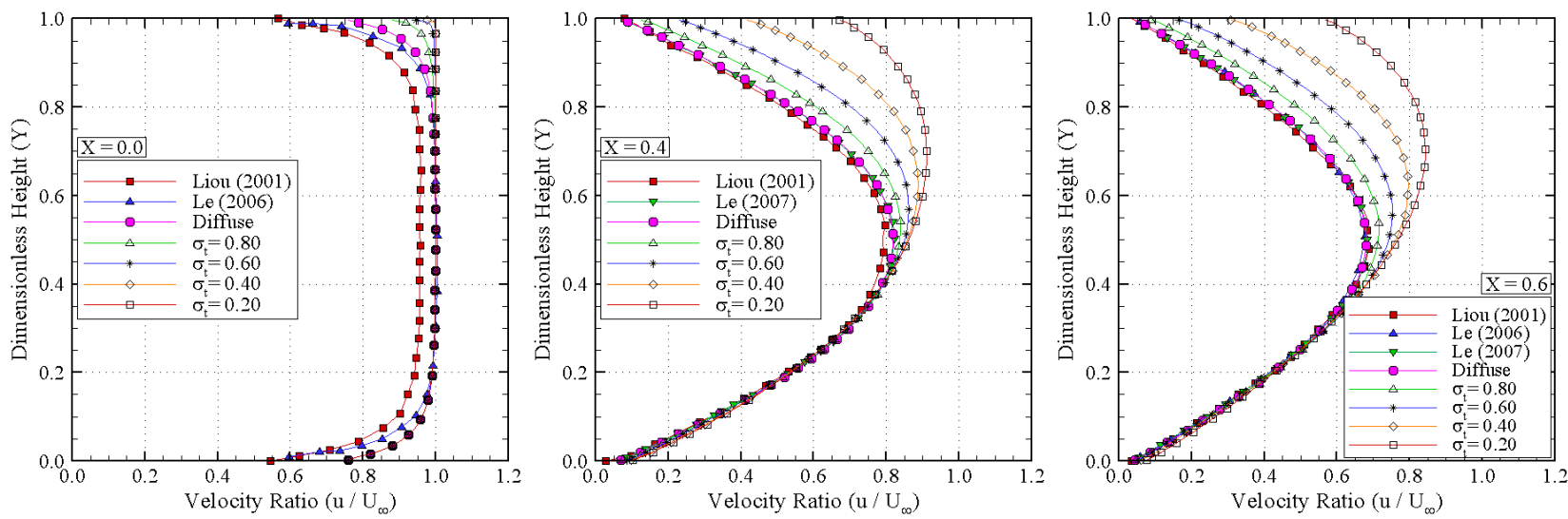

Figure 4. Velocity ratio $\left(u / U_{\infty}\right)$ profiles along the microchannel as a function of the tangential accommodation coefficient $\sigma_{t}$ for section $X$ of 0.0 (left), 0.4 (middle) and 0.6 (right).
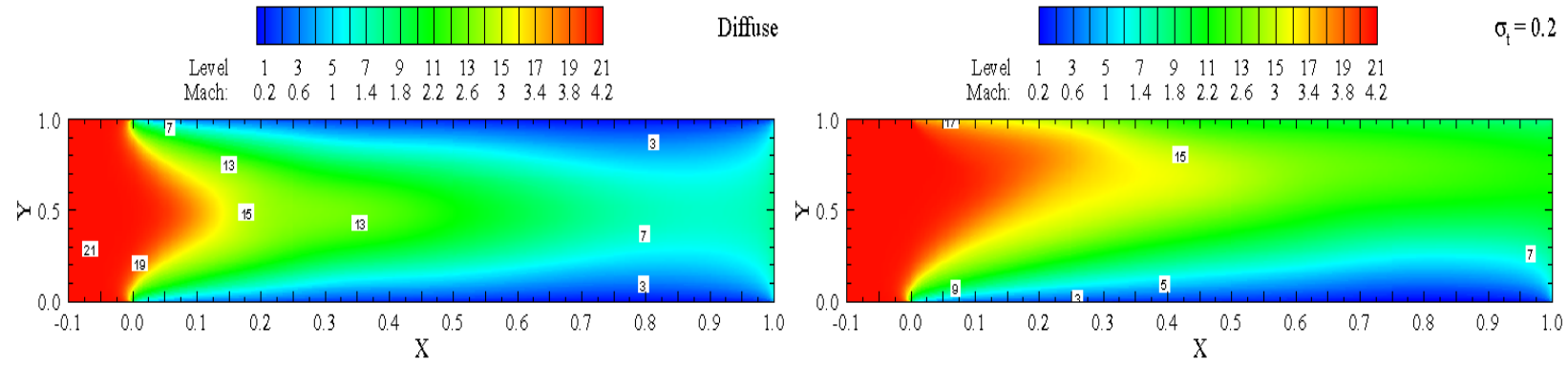

Figure 5. Mach number contour map along the microchannel for the diffuse case (left) and for the $\sigma_{t}=0.2$ case (right).

velocity ratio profiles. It is observed that a reduction in the tangential accommodation coefficient $\sigma_{t}$ from 1.0 to 0.2 results in an asymmetry in the velocity ratio profiles, not only along the entrance region but also along the fully developed region. It is also seen that the primary peak value for the velocity ratio, located at the centerline of the channel for the diffuse case, moves to the upper-most portion of the channel with decreasing $\sigma_{t}$. This is explained by the fact that the smaller the $\sigma_{t}$ is the smaller the viscous action at the vicinity of the upper surface. Consequently, the development of the boundary layers along the lower and upper surfaces is different from one to another. Of great significance in this set of plots is the slip velocity. It is quite apparent that slip velocity increases on the upper surface with decreasing $\sigma_{t}$. 
In an effort to emphasize points of interest, the distribution of Mach number in the whole microchannel is demonstrated in Fig. 5 for the diffuse reflection case and for the $\sigma_{t}=0.2$ case. The Mach number contour maps for the whole flowfield confirm that flow is affected by a reduction of $\sigma_{t}$ in almost all parts of the flowfield. For the diffuse case, the leading-edge shocks are thick, and the oblique shock angles, measured from the channel wall, are large. In addition, it is observed that detached bow shocks are formed in front of the leading edges because of the viscous boundary layers. Moreover, the Mach number varies from supersonic flow to sonic flow at the outflow boundary. For the $\sigma_{t}=0.2$ case, a different flow patterns is noticed. The leading-edge shocks are different from one to another. The higher Mach number region at the entrance extends far downstream in the upper-most part of the channel. Furthermore, at the outflow boundary, the flow is supersonic in the half-upper part of and sonic in the half-lower part of the microchannel, except at the vicinity of the lower surface. Therefore, particular attention should be paid to the outflow boundary condition in the sense that it should handle both supersonic and subsonic conditions.

\subsection{Density Profiles}

The impact of normal $\alpha_{n}$ and tangential $\sigma_{t}$ accommodation coefficients on density profiles are displayed in Figs. 6 and 7, respectively, for three cross-sections along the microchannel. In this group of plots, density ratio refers to density $\rho$ normalized by the freestream density $\rho_{\infty}$. According to these plots, for the diffuse reflection case, it is noticed that the profiles are symmetric with respect to the microchannel centerline, as expected. At the vicinity of the walls, density ratio increases downstream along the microchannel. In the first half part of the microcannel, density ratio profiles present twin points corresponding minimum values. After that, it seems that these points merge in the second half part of the microchannel.

As indeed is clear from these plots, partial surface accommodation affects density ratio profiles. It is seen that a reduction in the normal accommodation coefficient $\alpha_{n}$ from 1.0 to 0.2 causes a mild changes in the density ratio profiles, more precisely at the vicinity of both plate surfaces. Broadly speaking, density ratio increases at the lower plate and decreases at the upper plate with decreasing $\alpha_{n}$. In contrast, a reduction in the tangential accommodation coefficient $\sigma_{t}$ from 1.0 to 0.2 results in significant changes in the density ratio profiles as the flow develops downstream along the microchannel. It is somewhat surprising to find out that density ratio decreases not only near the upper wall but also near the lower wall. It is important to recall that a reduction in $\sigma_{t}$ is only imposed on the upper wall.

In order to bring out the essential features associated with density ratio behavior, contour map for density ratio distribution in the whole microchannel is depicted in Fig. 8 for the diffuse reflection case and for the $\sigma_{t}=0.2$ case. Similar to Mach number contour maps, density contour maps for the whole flowfield confirm that the flow is affected by a reduction of $\sigma_{t}$ in almost all parts of the flowfield. For the diffuse case, the shock waves generated from the two leading edges of the plates intersect one to another, and then extend to the wall. As a result, this cause the formation of islands of low density near the walls in the first half part of the microchannel. Behind the intersection, a region of high density appears. Afterwards, in the second half part of the microchannel, the density ratio dramatically increases very close to the walls. For the $\sigma_{t}=0.2$ case, a completely different flow patterns is observed. It is seen that a shock wave emanates only from the leading edge of the lower plate. The shock layer is much thicker than that for the diffuse case. The reason for that is because no shock wave is formed from the leading edge of the upper plate. As a result, a large island of low density is identified near the lower wall.
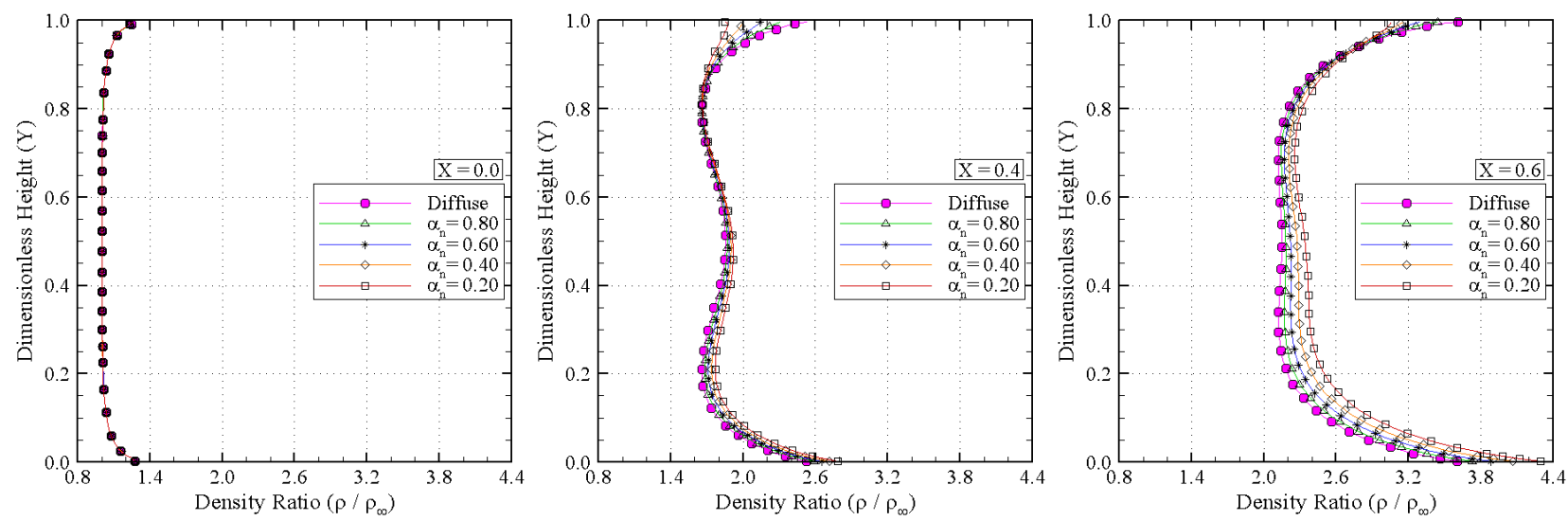

Figure 6. Density ratio $\left(\rho / \rho_{\infty}\right)$ profiles along the microchannel as a function of the normal accommodation coefficient $\alpha_{n}$ for section $X$ of 0.0 (left), 0.4 (middle) and 0.6 (right). 

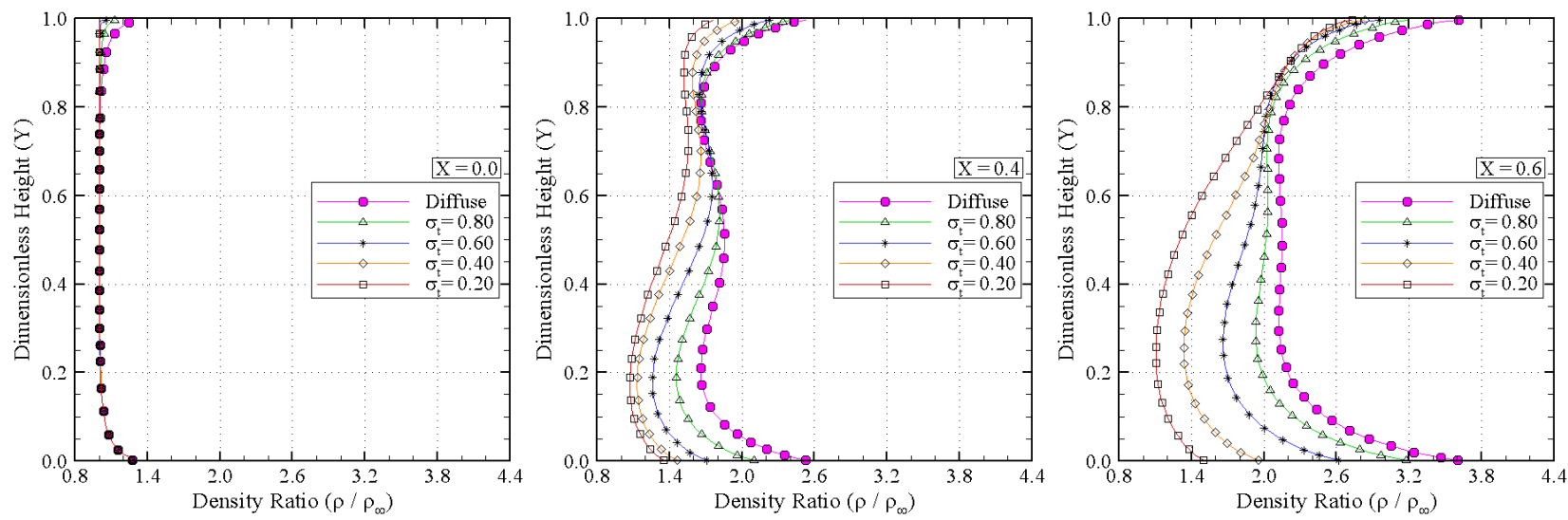

Figure 7. Density ratio $\left(\rho / \rho_{\infty}\right)$ profiles along the microchannel as a function of the tangential accommodation coefficient $\sigma_{t}$ for section $X$ of 0.0 (left), 0.4 (middle) and 0.6 (right).
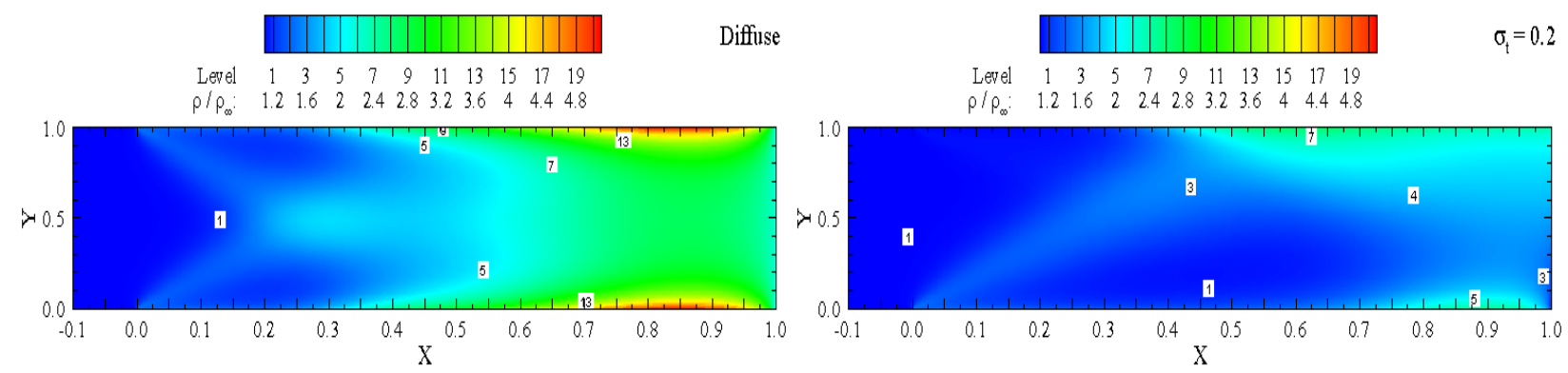

Figure 8. Density ratio $\left(\rho / \rho_{\infty}\right)$ contour map along the microchannel for the diffuse case (left) and $\sigma_{t}=0.2$ case (right).

By examining the density profiles depicted in Figs. 6, 7 and 8, it is observed that the normal and tangential accommodation coefficients produce different behaviors. These differing behaviors are caused basically by differences in the velocity distributions of the scattered molecules. When $\alpha_{n}$ and $\sigma_{t}$ are equal to 1 , the molecules reflect diffusely, which have a Maxwellian velocity distribution based on the surface temperature. For a cold wall, the diffusely reflected molecules remain at the vicinity of the body surface longer and result in a density buildup near the wall. Nevertheless, when $\alpha_{n}$ or $\sigma_{t}$ are different from 1, the CLL model provides a much complex description of the velocity distribution of scattered molecules in which the overall mean velocity and mean scattered angle are complex functions of the incoming velocity, surface temperature, and the normal and tangential accommodation coefficients. For the surface accommodation coefficients less than one investigated, the CLL model gives a somewhat different mean velocity of scattered molecules and a distribution of scattered molecules tending to the specular angle (see Fig. 1). Consequently, molecules do not remain near the body surface as long as with the diffuse reflection model, and low density occurs close to the body surface.

\subsection{Pressure Profiles}

Effects of normal $\alpha_{n}$ and tangential $\sigma_{t}$ accommodation coefficients on pressure profiles are illustrated in Figs. 9 and 10, respectively, for three cross-sections along the microchannel. In this set of diagrams, pressure ratio represents the pressure $p$ normalized by the freestream pressure $p_{\infty}$.

Based on Figs. 9 and 10, it is quite apparent that pressure ratio increases significantly as the flow develops downstream along the microchannel. Nevertheless, it is very encouraging to observe that, compared to the pressure ratio for the diffuse reflection case, pressure ratio increases with changes in the normal accommodation coefficient $\alpha_{n}$. Conversely, pressure ratio decreases with a reduction in the tangential accommodation coefficient $\sigma_{t}$ from 1.0 to 0.2 .

Pressure behavior along the centerline may be compared qualitatively with results obtained from the one-dimensional compressible flow solution in constant-area ducts with friction, i.e., the Fanno solution (Shapiro, 1954). For the particular case of pressure, the governing equations given by the Fanno solution are as following, 

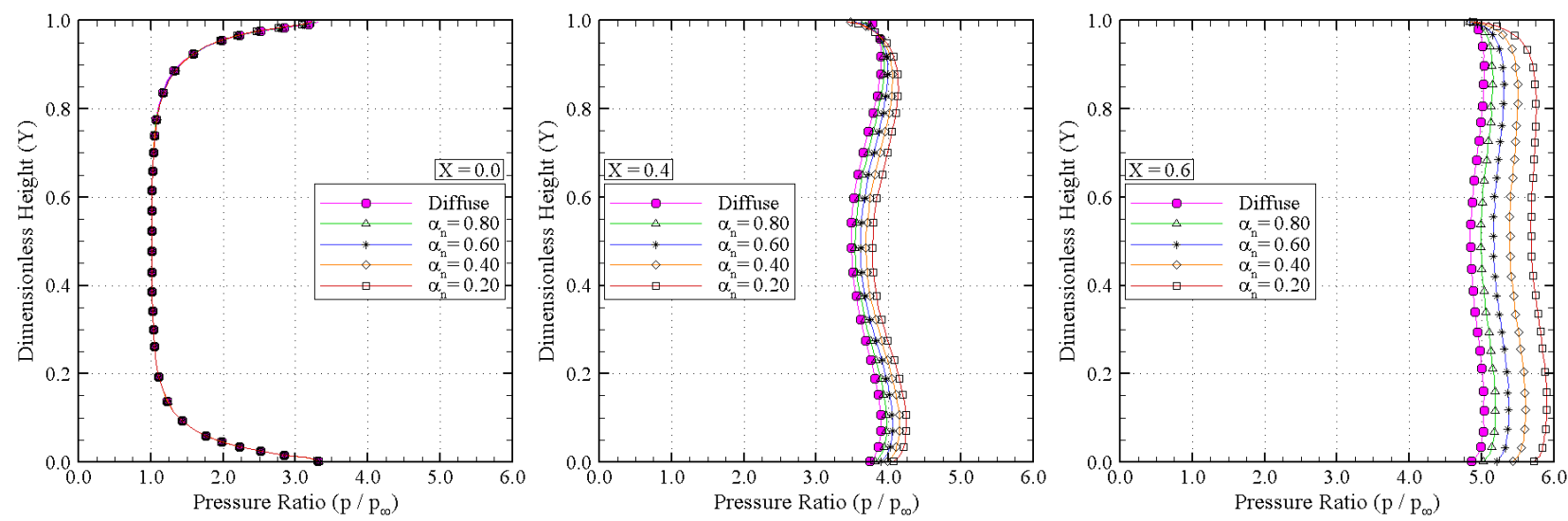

Figure 9. Pressure ratio $\left(p / p_{\infty}\right)$ profiles along the microchannel as a function of the normal accommodation coefficient $\alpha_{n}$ for section $X$ of 0.0 (left), 0.4 (middle) and 0.6 (right).
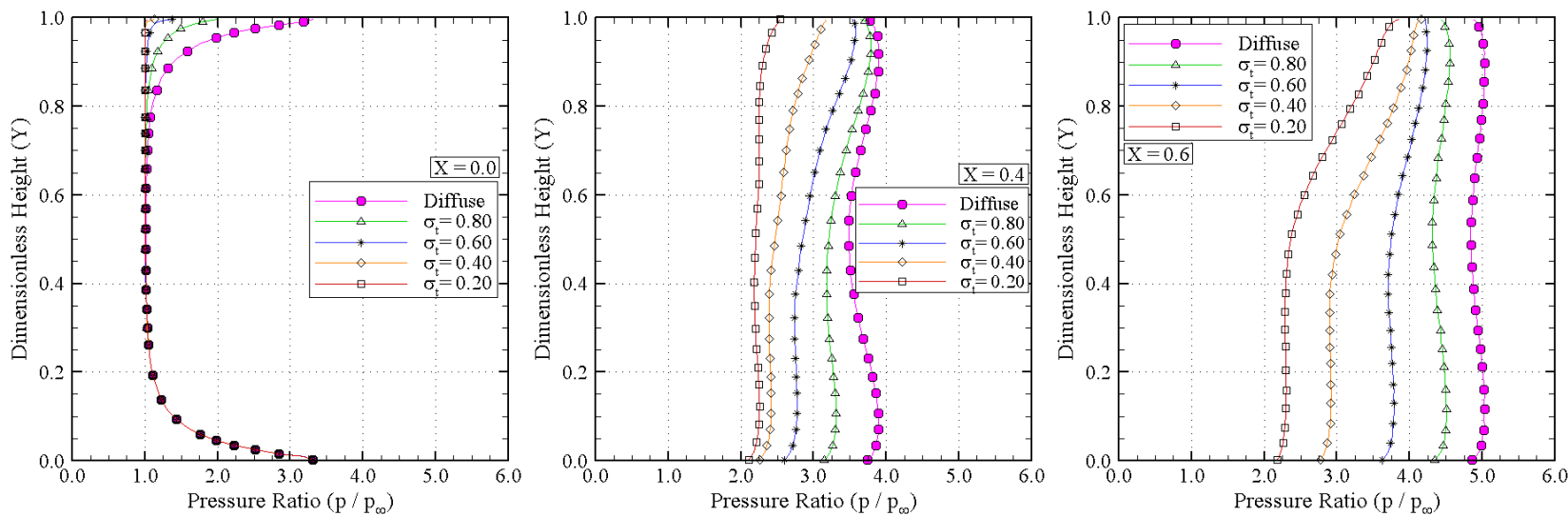

Figure 10. Pressure ratio $\left(p / p_{\infty}\right)$ profiles along the microchannel as a function of the tangential accommodation coefficient $\sigma_{t}$ for section $X$ of 0.0 (left), 0.4 (middle) and 0.6 (right).

$$
\begin{aligned}
& \frac{p_{2}}{p_{1}}=\frac{M_{1}}{M_{2}}\left[\frac{2+(\gamma-1) M_{1}^{2}}{2+(\gamma-1) M_{2}^{2}}\right]^{1 / 2} \\
& \frac{4 f d x}{D_{h}}=\frac{2}{\gamma M^{2}}\left(1-M^{2}\right)\left[1+\frac{(\gamma-1)}{2} M^{2}\right]^{-1} \frac{d M}{M}
\end{aligned}
$$

where $f$ is the friction coefficient, $D_{h}$ is the duct hydraulic diameter, $\gamma$ is the specific heat ratio, and subscripts 1 and 2 refer, respectively, to upstream and downstream $x$ stations along the duct.

Since the present flow is supersonic, $M>1$, then Eq. 3 can be rewritten as,

$$
\frac{d M}{d x}=-\eta f
$$

where $\eta=\eta\left(M, D_{h}, f, \gamma\right)$ and $\eta>0$.

As $f>0$, then Mach number decreases downstream along the duct, i.e., $M_{2}<M_{1}$. Therefore, from Eq. 2, one has that $p_{2}>p_{1}$.

Although shock waves were generated from the leading edges of the plates, results in the present account are consistent with the Fanno results in the sense that, in general, pressure increased and the Mach number decreased along the microchannel, and reached sonic conditions at the outflow boundary. 

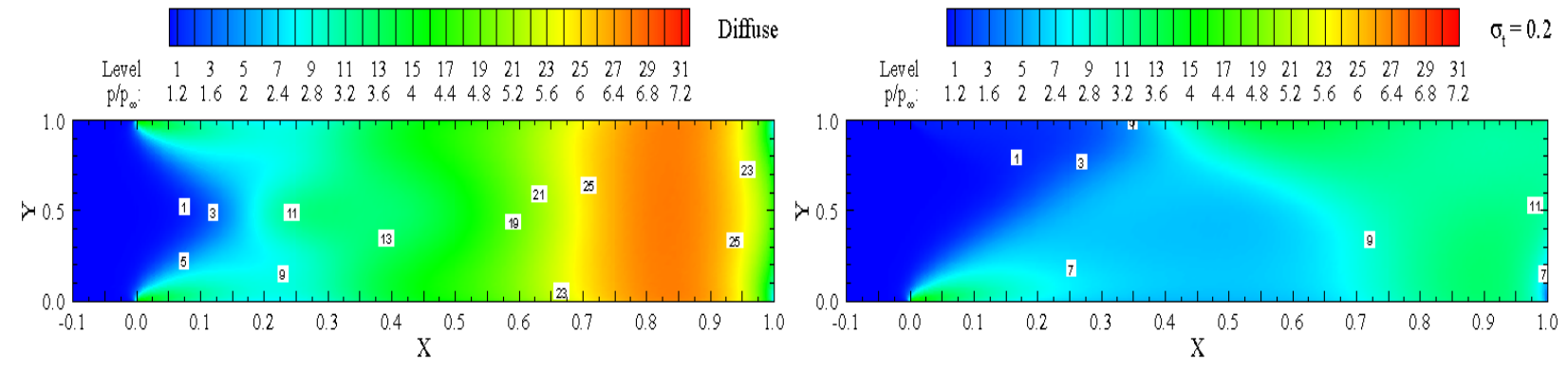

Figure 11. Pressure ratio $\left(p / p_{\infty}\right)$ contour map along the microchannel for the diffuse case (left) and $\sigma_{t}=0.2$ case (right).

Having a clear qualitative picture of the flow patterns associated with pressure profiles, it proves convenient to assess the overall performance of the pressure in the entire microchannel. In this manner, contour map for pressure ratio distribution is illustrated in Fig. 11 for the diffuse reflection case and for the $\sigma_{t}=0.2$ case.

\subsection{Kinetic Temperature Profiles}

Changes on kinetic temperature due to variations in the normal $\alpha_{n}$ and tangential $\sigma_{t}$ accommodation coefficients are presented in Figs. 12 and 13, respectively, for three cross-sections along the microchannel. In this group of plots, kinetic temperature ratio refers to the translational temperature $T_{t}$ normalized by the freestream temperature $T_{\infty}$. In addition, as a base of comparison, temperature profiles obtained by Liou and Fang (2001), Le and Hassan (2006) and Le and Esmail (2007) are also presented in these plots.

According to these plots, for the diffuse reflection case, it is noticed that temperature profiles are similar to the density profiles in the sense that the profiles are symmetric with respect to the microchannel centerline. it is seen that, at the vicinity of the walls, temperature ratio increases due to the viscous effects and, then decreases up to the centerline of the microchannel. In addition, the temperature ratio profiles present twin peaks values. After that, it seems that these peaks merge in the second half part of the microchannel. Based on the profile at section $X=0.6$, it is seen that the fully developed state is not reached yet.

It is also observed in these group of plots that partial surface accommodation affects temperature ratio profiles. It is seen that a reduction in the normal accommodation coefficient $\alpha_{n}$ from 1.0 to 0.2 causes a mild changes in the temperature ratio profiles, more precisely temperature ratio increases from the centerline to the upper surface. In contrast, a reduction in the tangential accommodation coefficient $\sigma_{t}$ from 1.0 to 0.2 result in significant changes in the temperature ratio profiles as the flow develops downstream along the microchannel. It is noticed that temperature ratio decreases not only near the upper wall but also near the lower wall.

Still referring to these plots, a good agreement is obtained as the temperature ratio profiles are compared with those presented by Le and Hassan (2006) and Le and Esmail (2007). However, at section $X$ of 0.4 and 0.6, a small difference
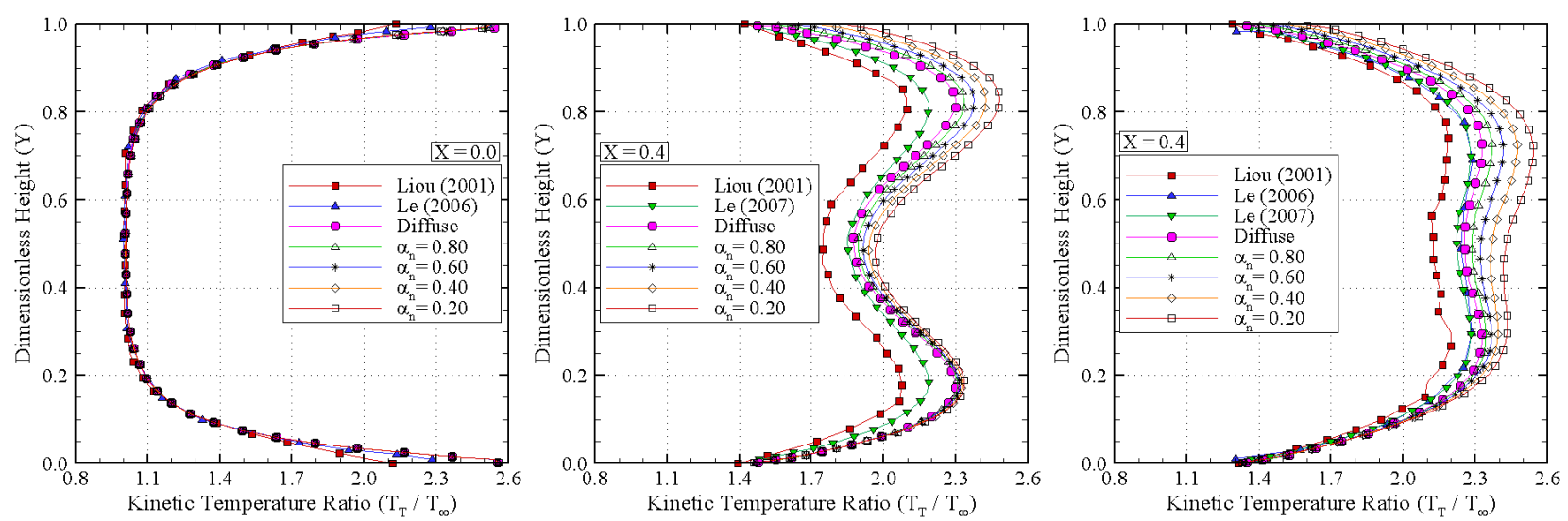

Figure 12. Kinetic temperature ratio $\left(T_{t} / T_{\infty}\right)$ profiles along the microchannel as a function of the normal accommodation coefficient $\alpha_{n}$ for section $X$ of 0.0 (left), 0.4 (middle) and 0.6 (right). 

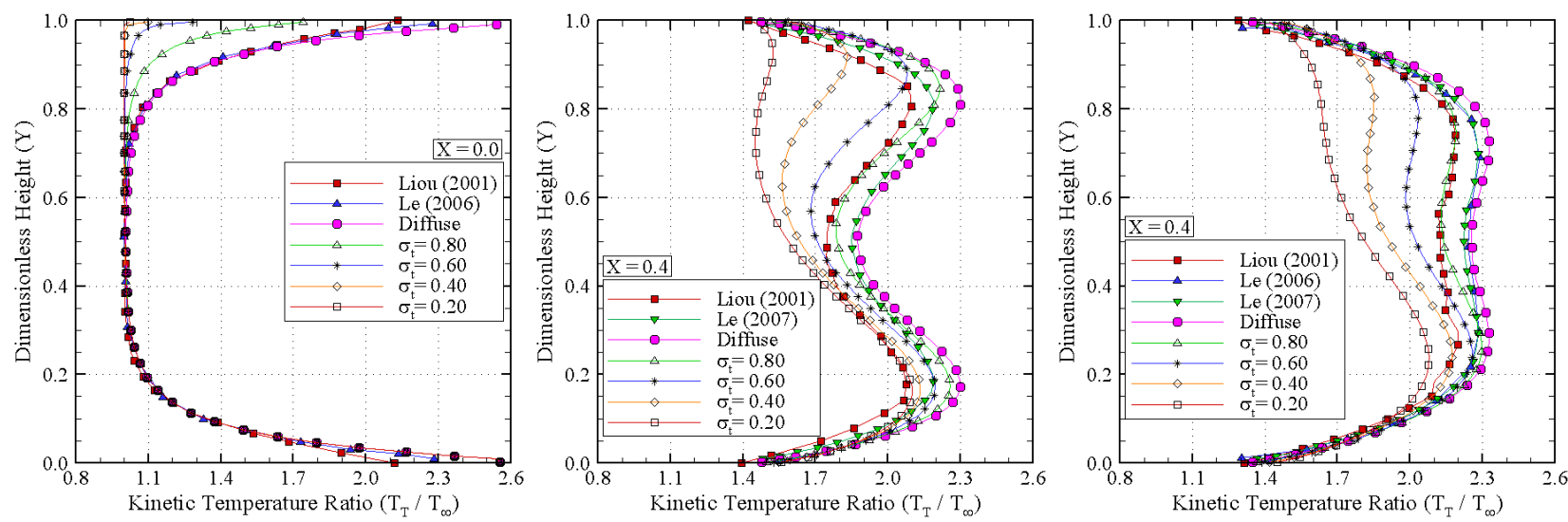

Figure 13. Kinetic temperature ratio $\left(T_{t} / T_{\infty}\right)$ profiles along the microchannel as a function of the tangential accommodation coefficient $\sigma_{t}$ for section $X$ of 0.0 (left), 0.4 (middle) and 0.6 (right).
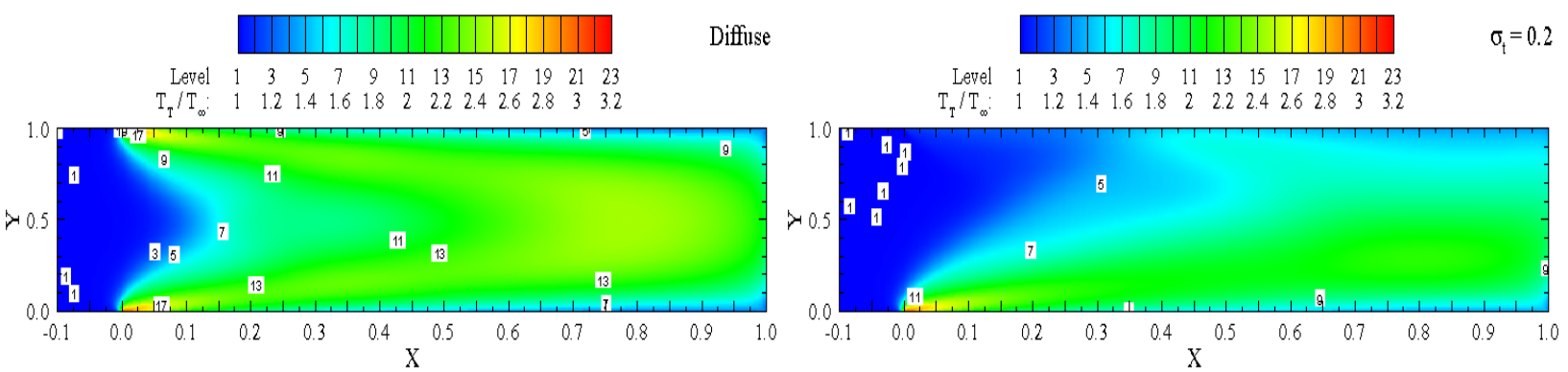

Figure 14. Translational temperature ratio $\left(T_{t} / T_{\infty}\right)$ contour map along the microchannel for the diffuse case (left) and $\sigma_{t}$ $=0.2$ case (right).

in the temperature profiles is observed as the solution is compared with DSMC results reported by Liou and Fang (2001).

Finally, at this point it is worth taking a close look at the temperature behavior in the entire microchannel. In doing so, contour map for translational temperature ratio distribution is displayed in Fig. 14 for the diffuse reflection case and for the $\sigma_{t}=0.2$ case.

\section{CONCLUDING REMARKS}

In the present work, a rarefied supersonic flow in a microchannel has been investigated by using the Direct Simulation Monte Carlo (DSMC) method. The simulations provided information concerning the nature of the incomplete surface accommodation effects in the primary properties for different combinations of normal and tangential accommodation coefficients on the upper surface. This parametric study was performed by changing separately the normal and tangential accommodation coefficients from 1.0 to 0.2 .

Profiles at different microchannel sections showed that normal and tangential accommodation coefficients provided an opposite influence under the primary proprieties. In general, primary proprieties showed more sensitivity to tangential accommodation coefficient changes. The analysis also showed that the microchannel lower region is sensitive to changes on the accommodation coefficients on the upper surface, and it is worthwhile mentioning that in some cases this sensitivity is greater than that observed at the upper region.

Among the present simulations, further calculations with different surface temperatures, freestream velocities or angles attack might be performed. Moreover, to simulate microchannels with different accommodation in the same surface can provide more insight into the sensitivity of primary proprieties to surface roughness.

\section{ACKNOWLEDGEMENTS}

The authors would like to thank the financial support provided by CNPq (Conselho Nacional de Desenvolvimento Científico e Tecnológico) under Grant No. 580249/2009-5. 


\section{REFERENCES}

Alexeenko, A. A., Gimelshein, S. F. and Levin, D. A., 2003. Reconsideration of Flows through Constriction Microchannels using the DSMC method. 36th AIAA Thermophysics Conference, AIAA Paper 2003-4009.

Barber, R. W. and Emerson D. R., 2002. The Influence of Knudsen Number on the Hydrodynamic Developments Length within Parallel Plate Micro-Channels. Advances Fluid Mechanics, Vol. IV, pp. 207-216.

Bird, G. A., 1981. Monte Carlo Simulation in an Engineering Context. In Fisher, S. S., ed., Progress in Astronautics and Aeronautics: Rarefied gas Dynamics, Vol. 74, part I, AIAA New York, pp. 239-255.

Bird, G. A., 1989. Perception of Numerical Method in Rarefied Gasdynamics. In Muntz, E. P., Weaver, D. P. and Capbell, D. H., eds., Rarefied Gas Dynamics: Theoretical and Computational Techniques, Vol. 118, Progress in Astronautics and Aeronautics, AIAA, New York, pp. 374-395.

Bird, G. A., 1994. Molecular Gas Dynamics and the Direct Simulation of Gas Flows Oxford University Press.

Borgnakke, C. and Larsen, P. S., 1975. Statistical Collision Model for Monte Carlo Simulation of Polyatomic Gas Mixture. Journal of Computational Physics, Vol. 18, No. 4, pp. 405-420.

Cai, C., and Boyd, I. D., 2007. Compressible Gas Flow Inside a Two-Dimensional Uniform Microchannel. Journal of Thermophysics and Heat Transfer, Vol. 21, No., pp. 608-615.

Cercignani, C. and Lampis, M., 1971. Kinetic Models for Gas-Surface Interactions. Transport Theory and Statistical Physics, Vol. 1, No. 2, pp. 101-114.

Gad-el-Hak, M., 1999. The Fluid Mechanics of Microdevices - The Freeman Scholar Lecture. Journal of Fluids Engineering, Vol. 121, No. , pp. 5-33.

Ho, C.-M., and Tai, Y.-C., 1996. MEMS and Its Applications for Flow Control. Journal of Fluids Engineering, Vol. 118, No. , pp. 437-447.

Ho, C.-M., and Tai, Y.-C., 1998. Micro-Electro-Mechanical Systems (MEMS) and Fluid Flows. Annual Review of Fluid Mechanics, Vol. 30, No. , pp. 579-612.

Kaplan, C. R., and Oran, E. S., 2002. Nonlinear Filtering for Low-Velocity Gaseous Microflows. AIAA Journal, Vol. 40, No. , pp. 82-90.

Karniadakis, G. E., Beskok, A., and Aluru, N., 2005. Microflows and Nanoflows, Fundamentals and simulation. Springer.

Le, M., and Hassan, I., 2006. Simulation of Heat Transfer in High Speed Microflows. Applied Thermal Engineering, Vol. 26, No. , pp. 2035-2044.

Le, M., and esmail, N., 2007. The Effects of Outlet Boundary Conditions on Simulating Supersonic Microchannel Flows using DSMC. Applied Thermal Engineering, Vol. 27, No., pp. 21-30.

Lilly, T. C., Ducan, J. A., and Nothnagel, S. L., 2007. Numerical and Experimental Investigation of Microchannel Flows with Rough Surfaces. Physics of Fluids, Vol. 19, No., pp. 106101:1-9.

Liou, W. W., and Fang, Y., 2001. Heat Transfer in Microchannel Devices using DSMC. Journal of MicroElectroMechanical Systems, Vol. 10, No., pp. 274-279.

Lord, R. G., 1991. Application of the Cercignani-Lampis Scattering Kernel to Direct Simulation Monte Carlo Method. Proceedings of the 17th International Symposium on Rarefied Gas Dynamics, edited by A. E. Beylich, Aachen, Germany, pp. 1427-1433.

O’Connor, L., 1992. MEMS: Microelectromechanical Systems. Mechanical Enginnering, Vol. 114, No. , pp. $40-47$.

Oh, C. K., Oran, E. S., and Sinkovits, R. S., 1997. Computations of High-Speed, High Knudsen Number Microchannel Flows. Journal of Thermophysics and Heat Transfer, Vol. 11, No. , pp. 497-505.

Sayegh, R., Faghri, M., Asako, Y., and Sundén, B., 2004. Direct Simulation Monte Carlo of Gaseous Flow and Heat Transfer in a Microchannel. Developments in Heat Transfer, Vol. 13, No., pp. 273-302.

Schaff, S. and Chambre P., 1958. Fundamentals of Gas Dynamics. Princeton University Press, Princeton, NJ.

Shapiro, A., 1954. The Dinamics and Thermodynamics of Compressible Fluid Flow. Ronald, Vol. II, New York, NI.

Sen, M., Wajerski, D., and Gad-al-Hak, M., 1996. A Novel Pump for MEMS Applications. Journal of Fluids Engineering, Vol. 118, No. , pp. 624-627.

Titov, E., Levin D., and Rogazinsky, S.V., 2007. Analyses of Numerical Errors in the Kinetic Modeling of Microthruster Devices. Journal of Thermophysics and Heat Transfer, Vol. 21, No. , pp. 616-622.

\section{Responsibility notice}

The authors are the only responsible for the printed material included in this paper 